\title{
Epidemiology of Pulmonary Embolism in Apulia from analysis of current data
}

\author{
A.M. Moretti1, S. Tafuri2, D. Parisi2, C. Germinario2
}

\begin{abstract}
Epidemiology of Pulmonary Embolism in Apulia from analysis of current data. A.M. Moretti, S. Tafuri, D. Parisi, C. Germinario.

Background and aims. Pulmonary embolism (PE) is a relatively common cardiovascular emergency: present evidence suggests that $\mathrm{PE}$ is the third most acute cardiovascular disease after cardiac ischemic syndromes and stroke. The aim of this study is to evaluate hospital admissions for PE in the Apulia Region of Italy in the period 2001-2007 through an analysis of the Apulia Region hospital patient discharge database.

Methods. Patients were selected on the basis of admissions between 01/01/2001 and 31/12/2007 with ICD-9-CM code of 415.11 (Iatrogenic pulmonary embolism and infarction) or 415.19 (Other pulmonary embolism and infarction) as principal or secondary diagnosis.
\end{abstract}

\begin{abstract}
Results. The number of patients selected from the database was 4,303 . The raw annual admission data shows an increasing trend from $13.9 \times 100,000$ residents in 2001 to $18.9 \times 100,000$ residents in 2007 . The average patient age was 68.7 years and $59 \%$ were females and $41 \%$. There were 470 deaths in hospital $(10.9 \%$ of patients).

Conclusions. $\mathrm{PE}$ is associated with much health care and a substantial economic burden, yet many $P E$ and general venous thromboembolism (VTE) events are preventable. It remains the responsibility of individual hospitals to identify specific areas in which they can improve their VTE prophylaxis rates to obtain positive results from the reporting initiatives and incentive programs.
\end{abstract}

Monaldi Arch Chest Dis 2010; 73: 1, 18-24.

Key words: Pulmonary embolism, Epidemiology, Health information systems, Patient safety.

1 Pneumology Department, Policlinico General Hospital, Bari,

2 Department of Biomedical Sciences, Hygiene Section, University of Bari, Apulia Regional Epidemiological Observatory, Bari, Italy.

Correspondence: Dr Annamaria Moretti, Pneumology Department, Policlinico General Hospital, P.zza G. Cesare 11, 70124 Bari, Italy; e-mail: moretti.am@alice.it

\section{Introduction}

Pulmonary embolism (PE) is a relatively common cardiovascular emergency: present evidence suggests that PE is the third most acute cardiovascular disease after cardiac ischemic syndromes and stroke [1].

Although PE and deep vein thrombosis (DVT) are two clinical presentations of venous thromboembolism (VTE) and, in most cases, PE is a consequence of DVT, PE has features that are distinct from DVT [2]. The risk of death related to the initial acute episode of recurrent PE is greater in patients diagnosed with $\mathrm{PE}$ than in those diagnosed with DVT [3]. According to prospective cohort studies, the acute case fatality rate for PE ranges from 7 to $11 \%$ [4].

PE can occur in patients without any identifiable patient-related predisposing factors. In fact, in the International Cooperative Pulmonary Embolism Registry the proportion of patients with idiopathic or unproved PE was about $20 \%$, but it is usual to identify one or more of the predisposing factors which include age, history of previous VTE, active cancer, neurological disease with extremity paresis, medical disorders causing prolonged bed rest, such as heart or acute respiratory failure, and congenital or acquired thrombophilia, hormone replacement therapy and oral contraceptive therapy [5].

In the United States, the prevalence of PE among hospitalised patients, according to data collected between 1979 and 1999 was $0.4 \%$, while 40-53 per 100,000 persons were diagnosed with PE per year [6].

The corresponding figures for Europe are not available, but various surveys have been performed among both hospitalised and non-hospitalised populations. A survey carried out in France in a defined population of 342,000 inhabitants shows the incidence of PE at 60 per 100,000 per year; many of whom had been hospitalized within the previous three months [7].

In Italy, analysis of current hospital admission data gives an estimated new PE incidence rate of $30-33$ per 100,000 inhabitants per year [8]. However the diagnosis of PE is difficult and can be missed because of its non specific clinical presentation. Several studies seem to indicate that the real incidence rate is higher than that shown by the data, especially those carried out on deceased patients which have shown that PE is still under-diagnosed in hospitalised patients $[9,10]$. An ad-hoc study performed within the Tuscany region of Italy 
showed a PE incidence rate of around 100 per 100,000 inhabitants per year [1].

The aim of this study is to evaluate hospital admissions for PE in the Apulia Region of Italy in the period 2001-2007.

\section{Methods}

The analysis of hospital admission and inhospital death was carried out using the Apulia Region hospital patient discharge database for the years 2001-2007, selecting those patients with an ICD-9-CM code of 415.11 (Iatrogenic pulmonary embolism and infarction) or 415.19 (Other pulmonary embolism and infarction) as principal diagnosis or one of the five secondary diagnoses.

Comorbidities of patients with a principal diagnosis of PE were ascertained from the codes recorded in the secondary diagnoses. When PE was a secondary diagnosis, the principal diagnosis was also identified.

Lethality-hospital was calculated as a proportion of deaths of total patients admitted with a diagnosis of pulmonary embolism.

For calculation purposes, the number of residents in Apulia was taken as the population resident at 01/01/2004 (data from the Italian National Statistics Office - ISTAT).

A linear regression model was used to analyse trends and the differences in trends were calculated utilisng the Chi square test for trend, with a value of $p<0.05$ considered significant. The analysis was carried out with the statistical software Epi-Info 6.00 (public domain software - CDC Atlanta, Georgia; WHO Geneva, Switzerland).

\section{Results}

The hospitals in the Apulia region have 18,844 beds, of which 18,522 are for acute cases.
Every year, 870.000 patients were hospitalised in Apulia.

The number of patients with a diagnosis of PE selected from the database was 4,303. The raw annual hospital admission data shows an increasing trend from 13.9 per 100,000 residents in 2001 to 18.9 per 100,000 residents in 2007; this increasing trend was at the limit of statistical significance $\left(r^{2}=0.6 ; p=0.05\right.$; table 1$)$.

For males $(41 \%)$, the hospitalisation rate per 100,000 increased from 11.2 in 2001 to 15.7 in 2007 , while for females $(59 \%)$ the rate per 100,000 increased from 16.5 to 21.8 in the same period. No significant differences in hospital admission were observed between the sexes (chi-square for linear trend: $0.04 ; p=0.83$ ).

More than half of the patients were over 71 years of age (table 2). The average patient age for the period relating to the study was 68.7 years, with

Table 1. - Number of admissions and raw hospitalisation rates for pulmonary embolism per 100,000 residents. Apulia 2001-2007

\begin{tabular}{lcc}
\hline Year & Admissions & $\begin{array}{c}\text { Raw hospitalization } \\
\text { rate per 100.000 }\end{array}$ \\
\hline 2001 & 563 & 13.9 \\
\hline 2002 & 559 & 13.8 \\
\hline 2003 & 621 & 15.4 \\
\hline 2004 & 549 & 13.6 \\
\hline 2005 & 622 & 15.4 \\
\hline 2006 & 626 & 15.5 \\
\hline 2007 & 763 & 18.9 \\
\hline
\end{tabular}

Table 2. - Number of admissions and raw hospitalization rates for pulmonary embolism per 100,000 residents by age range and sex. Apulia 2001-2007

\begin{tabular}{|c|c|c|c|c|c|c|}
\hline \multirow[b]{2}{*}{$\begin{array}{l}\text { Age } \\
\text { range }\end{array}$} & \multicolumn{2}{|c|}{$\mathbf{M}$} & \multicolumn{2}{|c|}{$\mathbf{F}$} & \multicolumn{2}{|c|}{ Total } \\
\hline & $\mathbf{N}^{\circ}$ & $\begin{array}{c}\text { Rate } \\
\text { x100.000 } \\
\text { residents }\end{array}$ & $\mathbf{N}^{\circ}$ & $\begin{array}{c}\text { Rate } \\
\text { x100.000 } \\
\text { residents }\end{array}$ & $\mathbf{N}^{\circ}$ & $\begin{array}{c}\text { Rate } \\
\text { x100.000 } \\
\text { residents }\end{array}$ \\
\hline [0-10] & 3 & 1.3 & 0 & - & 3 & 0.7 \\
\hline [11-20] & 25 & 9.8 & 14 & 5.8 & 39 & 7.9 \\
\hline [21-40] & 125 & 20.5 & 148 & 24.1 & 273 & 22.3 \\
\hline [41-60] & 315 & 63.7 & 367 & 69.8 & 682 & 66.8 \\
\hline [61-70] & 415 & 221.7 & 479 & 224.5 & 894 & 223.2 \\
\hline [71-80] & 585 & 449.5 & 861 & 492.4 & 1446 & 474.1 \\
\hline$[>80]$ & 298 & 624.8 & 668 & 771.8 & 966 & 719.6 \\
\hline
\end{tabular}


a progressive and linear increase from 66.5 in 2001 to 70.7 in 2007 ; $\left(r^{2}=0.86 ; p<0.01\right)$ (not shown).

Pulmonary embolism was the principal diagnosis in $66.9 \%\left(\mathrm{n}^{\circ}=2881\right)$ of cases and a secondary diagnosis in $33.1 \%\left(n^{\circ}=1419\right)$ of cases (in 3 cases it was both primary and secondary diagnosis and therefore discarded). There were 299 (7\%) records with a principal diagnosis of PE with no secondary diagnosis.

The most frequent secondary diagnoses in patients with a primary diagnosis of PE were: diseases of the circulatory system particularly arterial hypertension, cardiac dysrhythmias and phlebitis; diseases of the respiratory system particularly chronic obstructive pulmonary disease; and endocrine disorders (table 3 ).

In patients with a secondary diagnosis of $\mathrm{PE}$, the most frequent primary diagnoses were: diseases of the circulatory system particularly ischemic heart disease, cardiac dysrhythmias and thrombosis; diseases of the respiratory system particularly chronic obstructive pulmonary disease; neoplasms; injuries particularly fractures (table 4).

Most admissions for pulmonary embolism were in General Medicine ( $\mathrm{n}=1202,27.9 \%)$, Cardiology $(n=956,22,2 \%)$, Pneumology $(n=492,11.4 \%)$ and Coronary Intensive Care Units $(n=479,11.1 \%$; table 5).

There were 470 in-hospital deaths $(10.9 \%$ of patients) during the period under study, with an average age of 73.5 years; $195(41.5 \%)$ were males and $275(58.5 \%)$ females. There is a rising trend in the number of in-hospital deaths per year (figure 1).

Most in-hospital deaths occurred in patients admitted to General Medicine $(21.9 \%, n=103)$, Intensive Care $(21.1 \%, \mathrm{n}=99)$ and Coronary Intensive Care Units $(15.1 \%, \mathrm{n}=71)$ (table 6). Lethality was highest in Intensive Care, Neurology, Cardiac Surgical ward and Pediatric Nephrology. In the period studied, the last 2 wards had 5 and 1 patients with a PE diagnosis (table 6).

\section{Discussion}

With a lack of standardised tools to evaluate pulmonary embolism, hospitalisation records can be useful for epidemiologic analysis. The records are systematically compiled by medical personnel and are subject to quality controls at various levels (hospital trust, regional, ministerial), however they are also dependent on the quality of the diagnosis and its coding. Clinical signs and symptoms allow the physician to formulate only a pre-test probability of a patient having pulmonary embolism (the clinical probability), they are insufficient in themselves to either diagnose or rule out the condition [11]. Laboratory testing and imaging are thus required in all patients with suspected pulmonary embolism [12] but there are a large number of diagnostic tests and strategies available, with varying diagnostic value. Ventilation perfusion lung scan, spiral computed tomography and ultrasonography of leg veins have shown positive likelihood ratios for confirmation of PE [13], but hospital pa-
Table 3. - Secondary diagnoses of patients with a primary diagnosis of pulmonary embolism. Apulia 2001-2007

\begin{tabular}{|c|c|}
\hline Diagnosis & $\mathbf{N}^{\circ}$ \\
\hline Infectious and parasitic diseases & 82 \\
\hline Neoplasms & 285 \\
\hline $\begin{array}{l}\text { Endocrine, nutritional and metabolic diseases, } \\
\text { and immunity disorders }\end{array}$ & 691 \\
\hline Disorders of thyroid gland & 90 \\
\hline Diseases of the blood and blood-forming organs & 155 \\
\hline Mental disorders & 57 \\
\hline Diseases of the nervous system and sense organs & 106 \\
\hline Diseases of the circulatory system & 3245 \\
\hline Arterial hypertension & 908 \\
\hline Cardiac dysrhythmias & 490 \\
\hline Phlebitis and thrombophlebitis & 465 \\
\hline Ischemic heart disease & 264 \\
\hline Embolism and thrombosis & 217 \\
\hline Heart failure & 162 \\
\hline Diseases of arteries, arterioles, and capillaries & 123 \\
\hline Diseases of pulmonary circulation & 111 \\
\hline Diseases of the respiratory system & 906 \\
\hline Chronic obstructive pulmonary disease & 447 \\
\hline Diseases of the digestive system & 220 \\
\hline Diseases of the genitourinary system & 259 \\
\hline $\begin{array}{l}\text { Complications of pregnancy, childbirth, } \\
\text { and the puerperium }\end{array}$ & 6 \\
\hline Diseases of the skin and subcutaneous tissue & 31 \\
\hline $\begin{array}{l}\text { Diseases of the musculoskeletal system } \\
\text { and connective tissue }\end{array}$ & 103 \\
\hline Congenital anomalies & 14 \\
\hline Symptoms, signs, and ill-defined conditions & 202 \\
\hline Injury and poisoning & 154 \\
\hline Fracture & 113 \\
\hline
\end{tabular}

Factors influencing health status and contact with health services

tient discharge records do not show which diagnostic tests have been carried out and consequently we cannot be sure of a correspondence between the diagnosis and the diagnostic algorithms recommended by the guidelines [14].

Hospital admission rates in Apulia seem to be lower than those found in surveys carried out in the USA [6] and in France [7]. The present study shows an increasing trend in hospitalisation for this pathology. This, together with a strategic regional policy of reducing hospital admissions, is indicative of a real increase in its incidence. 
Table 4. - Primary diagnosis of patients with a secondary diagnosis of pulmonary embolism. Apulia 2001-2007

\begin{tabular}{lc}
\hline Diagnosis & $\mathbf{N}^{\circ}$ \\
\hline Infectious and parasitic diseases & 14 \\
\hline Neoplasms & 187 \\
\hline
\end{tabular}

Endocrine, nutritional and metabolic diseases,

and immunity disorders

Diseases of the blood and blood-forming organs $\quad 17$

Mental disorders 5

Diseases of the nervous system and sense organs $\quad 27$

Diseases of the circulatory system 373

Ischemic Heart Disease $\quad 50$

Cardiac dysrhythmias $\quad 52$

Thrombosis

19

Diseases of the respiratory system 260

Diseases of the digestive system $\quad 86$

Diseases of the genitourinary system

44

Complications of pregnancy, childbirth,

and the puerperium

8

Diseases of the skin and subcutaneous tissue

3

Diseases of the musculoskeletal system

and connective tissue

69

Congenital anomalies

3

Symptoms, signs, and ill-defined conditions

113

Injury and poisoning

172

Fracture

144

Factors influencing health status and contact with health service

The low admission rate demonstrated in the Apulia Region could be related to the lack of awareness of the disease, which can be the cause of the low number of admissions and the level of misdiagnosis. It is very important to consider the diagnosis if there is any suspicion of pulmonary embolism. Prompt diagnosis and treatment can dramatically reduce the morbidity and mortality of the disease. Unfortunately, the diagnosis is often missed, because pulmonary embolism frequently causes only vague and non-specific symptoms. Failure to identify pulmonary embolism due to lack of diagnosis remains the last major problem confronting successful detection of pulmonary embolism. If the healthcare providers, patient, and family are not aware of the risk factors and common clinical presentations of pulmonary embolism, the diagnosis will be overlooked. More widespread education of practitioners and the pub-
Table 5. - Discharge ward for patients admitted with pulmonary embolism. Apulia 2001-2007

\begin{tabular}{|c|c|c|}
\hline Discharge ward & $\mathbf{N}^{\circ}$ & $\%$ \\
\hline General Medicine & 1202 & 27.9 \\
\hline Cardiology & 956 & 22.2 \\
\hline Pneumology & 492 & 11.4 \\
\hline Coronary Intensive Care & 479 & 11.1 \\
\hline Geriatrics & 218 & 5.1 \\
\hline Orthopedics trauma ward & 173 & 4.0 \\
\hline Intensive Care & 132 & 3.1 \\
\hline General surgery & 129 & 3 \\
\hline Long stay & 101 & 2.3 \\
\hline Physical medicine and rehabilitation & 65 & 1.5 \\
\hline Neurology & 60 & 1.4 \\
\hline Vascular surgery & 41 & 0.9 \\
\hline Infectious disease & 40 & 0.9 \\
\hline Urology & 32 & 0.7 \\
\hline Nephrology & 30 & 0.7 \\
\hline Neurosurgery & 25 & 0.6 \\
\hline Hematology & 23 & 0.5 \\
\hline Obstetrics and gynecology & 20 & 0.5 \\
\hline Oncology & 20 & 0.5 \\
\hline Chest surgery & 17 & 0.4 \\
\hline Endocrinology & 10 & 0.2 \\
\hline Gastroenterology & 8 & 0.2 \\
\hline Radiotherapy & 7 & 0.2 \\
\hline Ear nose and throat & 5 & 0.1 \\
\hline Cardiac Surgical ward & 4 & 0.1 \\
\hline Dentistry & 3 & 0.1 \\
\hline Psychiatry & 3 & 0.1 \\
\hline Pediatrics & 2 & 0.05 \\
\hline Oncologic radiotherapy & 2 & 0.05 \\
\hline Plastic surgery & 1 & 0.02 \\
\hline Ophthalmology & 1 & 0.02 \\
\hline Dermatology & 1 & 0.02 \\
\hline Pediatric nephrology & 1 & 0.02 \\
\hline
\end{tabular}




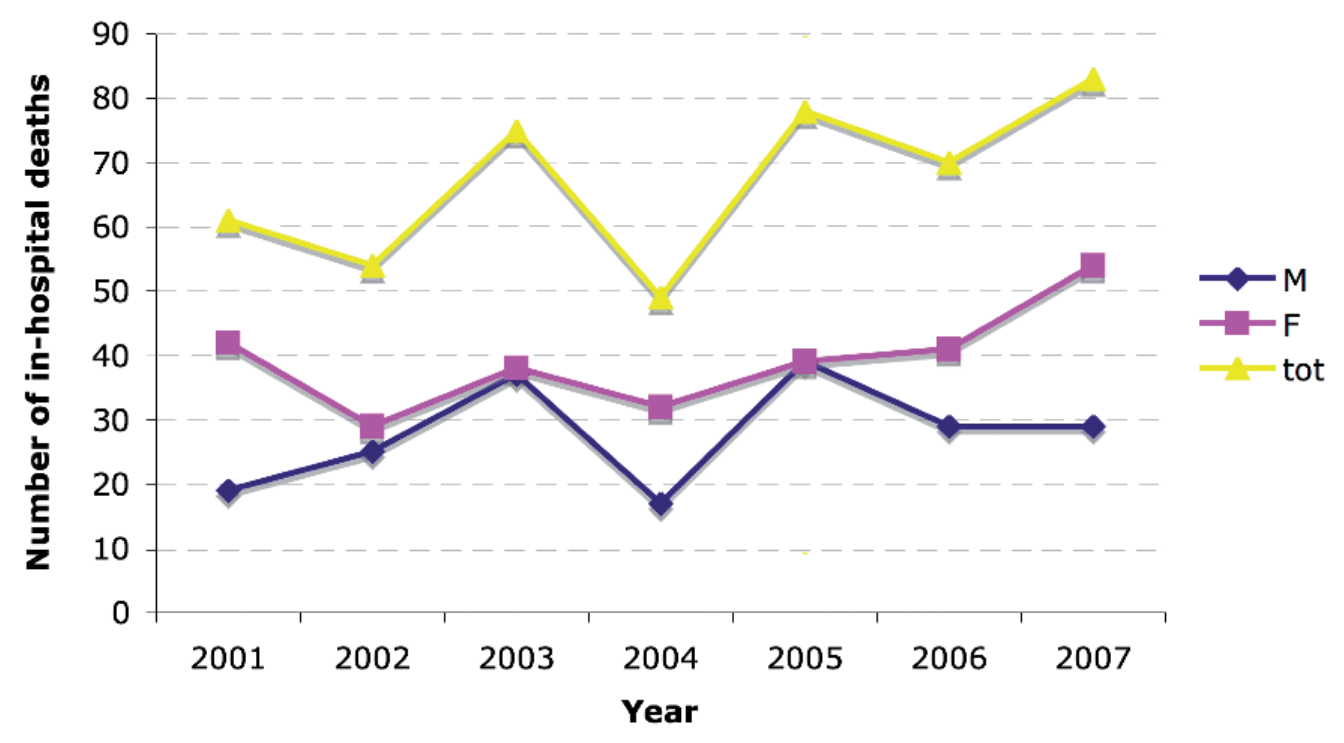

Fig. 1. - Number of in-hospital deaths for patients admitted with pulmonary embolism by sex and year of death. Apulia $2001-2007$.

lic about the potentially vague presentation of pulmonary embolism will raise awareness, improve vigilance, and result in fewer missed cases of this potentially fatal illness [15]. Hospital directors should improve the adoption of clinical protocols, especially in emergency care, to ensure correct assessment and management of the patient with suspect PE.

Patients who develop PE have frequent comorbidities. In a third of the cases evaluated, PE was a secondary diagnosis, however the discharge records do not allow us to ascertain whether the pathology was present at admission or if it arose later, also due to exposure of risk factors linked to hospitalisation. Additionally, an important proportion $(7 \%)$ of the records had no reported comorbidity, which can indicate the limitations of the recording system. These limitations and the high lethality of the pathology demonstrate the need to reinforce the risk assessment and management of the hospitalised patient and to adopt suitable primary and secondary prophylaxis protocols. Currently, three such protocols have been validated in a sufficient number of patients to be of clear utility: the Canadian, or Wells, score [16], the revised Genova score [17], and the PISA-PED score [18]. All these rules have potential pitfalls, and none is currently considered clearly better than the others. This situation has brought a certain lack of confidence in the application of this rigorous approach, likely limiting its use in clinical practice. Furthermore, the continuous attempts to modify, improve and simplify these scores make it difficult to complete and compare the prospective validation studies that are much needed for a widespread diffusion of this approach [19].

$\mathrm{PE}$ is associated with a substantial healthcare and economic burden, yet many PE and VTE in general events are preventable. Despite the availability of evidence-based guidelines detailing effective thromboprophylaxis strategies, the under- use and inappropriate prescribing of VTE prophylaxis is common. It remains the responsibility of individual hospitals to identify specific areas in which they can improve their VTE prophylaxis rates to obtain positive results from the reporting initiatives and incentive programs. If performance measures are to be met, all hospital departments will need to implement effective VTE prevention policies, including early risk assessment, appropriate prophylaxis prescribing, monitoring, and follow-up. Multifaceted, integrated initiatives involving risk assessment tools, decision support, electronic alert systems, and hospital-wide education, with a mechanism for audit and feedback, may help ensure that all healthcare professionals comply with VTE-prevention policies and initiatives [20].

In fact, the Commission on Accreditation of Healthcare Organizations considers the frequency of thromboembolic episodes in hospitalised patients an important safety indicator [21, 22].

The wide range of hospital wards where patients in the study have been treated, with eighty percent in four different wards (General medicine, Cardiology, Pneumology, Coronary Intensive Care Units) and the other twenty percent found in a grand variety of medical and surgical wards, allows us to see that the approach towards the pathology is not homogeneous, which could possibly cause a reduction in the levels of the appropriateness of treatment and patient safety, especially in as much as there is no defined protocol at hospital or regional level.

The Apulia Region hospital patient discharge database does not allow access either to the assessment procedures or to the prophylaxis protocols adopted, so no evaluation can be made of their appropriateness. Such an evaluation would require examination of the patients case notes which would be impracticable for epidemiological purposes. 


\begin{tabular}{|c|c|c|c|}
\hline Ward & $\mathbf{N}^{\circ}$ & $\%$ of total deaths & Lethality (\%) \\
\hline General Medicine & 103 & 21.9 & 8.6 \\
\hline Intensive Care & 99 & 21.1 & 75 \\
\hline Coronary Intensive Care & 71 & 15.1 & 14.8 \\
\hline Pneumology & 29 & 6.2 & 5.9 \\
\hline Geriatrics & 25 & 5.3 & 11.5 \\
\hline Cardiology & 23 & 4.9 & 2.4 \\
\hline General surgery & 23 & 4.9 & 17.8 \\
\hline Orthopedics trauma ward & 22 & 4.7 & 12.7 \\
\hline Neurology & 19 & 4 & 31.7 \\
\hline Long stay & 15 & 3.2 & 14.9 \\
\hline Infectious disease & 7 & 1.5 & 17.5 \\
\hline Neurosurgery & 7 & 1.5 & 28 \\
\hline Nephrology & 5 & 1.1 & 16.7 \\
\hline Cardiac Surgical ward & 4 & 0.9 & 100 \\
\hline Physical medicine and rehabilitation & 4 & 0.9 & 6.2 \\
\hline Hematology & 3 & 0.6 & 13 \\
\hline Oncology & 3 & 0.6 & 15 \\
\hline Urology & 3 & 0.6 & 9.4 \\
\hline Vascular surgery & 1 & 0.2 & 2.4 \\
\hline Dermatology & 1 & 0.2 & 100 \\
\hline Endocrinology & 1 & 0.2 & 10 \\
\hline Pediatric nephrology & 1 & 0.2 & 100 \\
\hline Obstetrics and gynecology & 1 & 0.2 & 5 \\
\hline
\end{tabular}

There is the need, within the regional health service, to adopt the monitoring of thromboembolic episodes to record risk factors, the risk class, the diagnostic procedures carried out and the treatment plans and so these can be integrated with the existing information channels.

Such monitoring and recording would give support to healthcare staff and allow the hospital management to evaluate the appropriateness and safety of the treatments. When adjusted for risk class and comorbidity, events such as "thromboembolic complications" and "in-hospital death due to thromboembolic complications", within the different clinical settings, could be properly evaluated against the quality of care given to determine which is best in class and which needs corrective intervention. Without this information, simple indicators such as frequency of events and lethality are limited in scope, not being able to take into consideration the case-mix within the hospital structure, so limiting the usefulness of the information here reported.

Clearly, the discharge database cannot give any information of incidence or death from pulmonary embolism which arise after hospital discharge of a patient. Such information can be obtained only through the adoption and upkeep of suitable information systems such as patient-files at primary care level and their data integration with the activities within secondary care. 


\section{References}

1. Giustini C, Di Ricco G, Marini C, Melillo E, Palla A. Pulmonary embolism: epidemiology. Chest 1995; 107 (1 Suppl): 3S-9S.

2. Dalen JE. Pulmonary embolism: what we learned since Virchow? Natural history, pathophysiology, and diagnosis. Chest 2002; 122: 1440-1456.

3. Murin S, Romano PS, White RH. Comparison of outcomes after hospitalization for deep venous thrombosis or pulmonary embolism. Thromb Haemost 2002; 88: 407-414.

4. Stein PD, Kayali F, Olson RE. Estimated case fatality rate of pulmonary embolism, 1979 to 1998 . Am J Cardiol 2004; 93: 1197-1199.

5. Goldhaber SZ, Visani L, De Rosa M. Acute pulmonary embolism: clinical outcomes in the International Cooperative Pulmonary Embolism Registry (ICOPER). Lancet 1999; 353: 1386-1389.

6. Stein PD, Beemath A, Olson RE. Trends in the incidence of pulmonary embolism and deep venous thrombosis in hospitalized patients. Am J Cardiol 2005; 95: 1525-1526.

7. Oger E. Incidence of venous thromboembolism: a community based study in Western France. EPI-GETBP Study Group. Thromb Haemost 2000; 83: 657-60.

8. Roncon L, Zonzin P, Giommi L, et al. The diagnostic and therapeutic procedures in pulmonary embolism: a survey in the Veneto Region. Cardiologia 1999; 44: 735-41.

9. Goldhaber SZ, Hennekens CH, Evans DA, Newton EC, Godleski JJ. Factors associated with correct antemortem diagnosis of major pulmonary embolism. Am J Med 1982; 73: 822-6.

10. Rubinstein I, Murray D, Hoffstein V. Fatal Pulmonary Emboli in Hospitalized Patients. An Autopsy study. Arch Intern Med 1988; 148: 1425-26.

11. Chunilal SD, Eikelboom JW, Attia J, et al. Does this patients have pulmonary embolism? JAMA 2003; 290: 2849-58.

12. Fedullo PF, Tapson VF. Clinical practice. The evalua- tion of suspected pulmonary embolism. $N$ Engl J Med 2003; 349: 1247-56.

13. Roy PM, Colombet I, Durieux P, Chatellier G, Sors H, Meyer G. Sistematic review and meta-analysis of strategies for the diagnosis of suspected pulmonary embolism. BMJ 2005; 331: 239.

14. Torbicki A, Perrier A, Konstantinides S, et al. Guidelines on the diagnosis and management of acute polmonary embolism. European Heart Journal 2008; 29: 2276-2315.

15. Goldhaber SZ. Diagnosis of Acute Pulmonary Embolism: Always Be Vigilant. Am J Med 2007; 120: 827828.

16. Wells PS, Anderson DR, Rodger M, et al. Derivation of a simple clinical model to categorize patients probabilità of pulmonary embolism: increasing the models utilità with the SimpliRED D-dimer. Thromb Haemost 2000; 83: 416-420.

17. Le Gal G, Righini M, Roy PM, et al. Prediction of pulmonary embolism in the emergency department: the revised Genova Score. Ann Intern Med 2006; 144: 165171.

18. Miniati M, Prediletto R, Formichi B, et al. Accuracy of clinical assessment in the diagnosis of pulmonary embolism. Am J Respir Crit Care Med 1999; 159: 864-871.

19. Celi A, Marconi L, Villani L, Palla A. The diagnosis of pulmonary embolism. Monaldi Arch Chest Dis 2009; 71: 47-53.

20. Amin AN, Deitelzweig SB. Optimizing the Prevention of Venous Thromboembolism: Recent Quality Initiatives and Strategies to Drive Improvement. Jt Comm J Qual Patient Saf 2009; 35: 558-564.

21. Joint Commission on Accreditation of Healthcare Organizations: National Library of Healthcare Indicators (1998) Health Plan and Network Editing http: //www.jcaho.com/accredited+organizations/behavioral+health+care/oryx/index.htm

22. Henderson KE, Recktenwald A, Reichley RM, et al. Clinical validation of the AHRQ postoperative venous thromboembolism patient safety indicator. Jt Comm J Qual Patient Saf 2009; 35: 370-6.

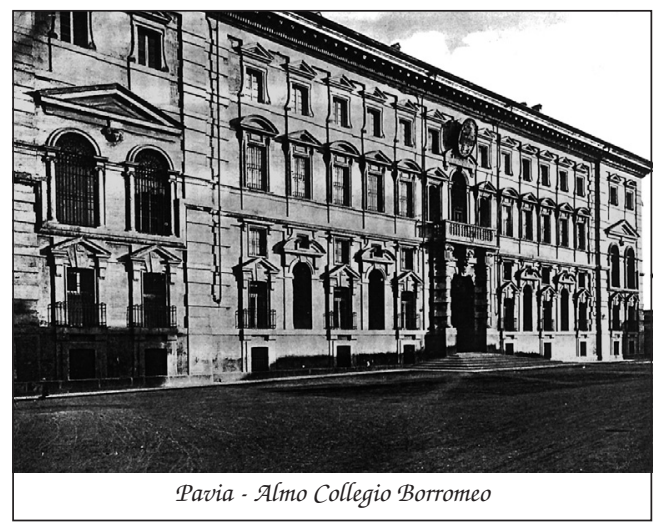

